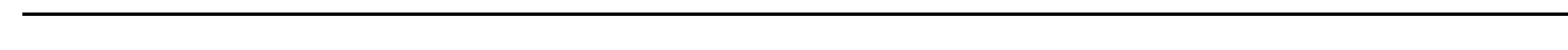

12 


\section{Infants with congenital nephrotic syndrome have comparable outcomes to infants with other renal diseases}

Stephanie Dufek ${ }^{1}$ (D) \&Elisa Ylinen ${ }^{2} \&$ Agnes Trautmann ${ }^{3} \&$ Harika Alpay ${ }^{4} \& G e m a$ Ariceta ${ }^{5} \&$ Christoph Aufricht ${ }^{6} \&$ J ustine Bacchetta ${ }^{7} \&$ Sevcan Bakkaloglu ${ }^{8} \&$ Aysun Bayazit ${ }^{9} \&$ Salim Caliskan ${ }^{10} \&$ Maria do Sameiro Faria ${ }^{11} \&$ Ismail Dursun ${ }^{12}$ \&M esiha Ekim ${ }^{13}$ \&Augustina J ankauskiene ${ }^{14}$ \&Günter Klaus ${ }^{15}$ \&Fabio Paglialonga ${ }^{16}$ \&Andrea Pasini ${ }^{17} \&$ Nikoleta Printza ${ }^{18} \&$ Valerie Said Conti ${ }^{19}$ \&Claus Peter Schmitt ${ }^{3} \&$ Constantinos Stefanidis ${ }^{20}$ \&Enrico Verrina ${ }^{21}$ \& Enrico Vidal ${ }^{22} \&$ Hazel Webb ${ }^{1} \&$ Argyroula Zampetoglou ${ }^{20} \&$ Alberto Edefonti ${ }^{16}$ \&Tuula Holtta ${ }^{2} \&$ Rukshana Shroff ${ }^{1} \& O n$ behalf of the ESPN Dialysis Working Group

Received: 3 September 2018 / Revised: 12 October 2018 / Accepted: 18 October 2018 / Published online: 29 October 2018

\# IPNA 2018

Background

Methods

Results 





